\title{
Role of PIM2 in allergic asthma
}

\author{
WEI DU ${ }^{1 *}$, TIANTIAN CHEN ${ }^{1 *}$, YINGMENG $\mathrm{NI}^{1}$, XIAOXIA HOU $^{1}$, YOUCHAO YU ${ }^{1}$, \\ QI ZHOU ${ }^{2}$, FANG WU ${ }^{3}$, WEI TANG ${ }^{1}$ and GUOCHAO SHI ${ }^{1}$ \\ ${ }^{1}$ Department of Pulmonary Medicine; ${ }^{2}$ Shanghai Key Laboratory for Bone and Joint Diseases, \\ Shanghai Institute of Orthopedics and Traumatology; ${ }^{3}$ Department of Geratology, Ruijin Hospital, \\ School of Medicine, Shanghai Jiao Tong University, Shanghai 200025, P.R. China
}

Received December 16, 2016; Accepted July 21, 2017

DOI: $10.3892 / \mathrm{mmr} .2017 .7499$

\begin{abstract}
T cell-associated inflammation, particularly type 2 inflammation, has an important role in asthma pathogenesis, which is suppressed by regulatory T cells (Tregs). Proviral integration site for Moloney murine leukemia virus 2 (PIM2), a member off the serine/threonine kinase family, promotes the growth and survival of $\mathrm{T}$ cells and influences the function of Treg cells. However, whether PIM2 affects asthma pathogenesis remains unclear. Peripheral blood mononuclear cells and Treg cells from asthmatic and healthy subjects were obtained, and the expression level of PIM2 was measured by reverse transcription-quantitative polymerase chain reaction and immunocytochemistry. In addition, BALB/c female mice sensitized and challenged by ovalbumin were used as an asthma model, and PIM2 inhibitor was injected during the challenge period to observe the effect of PIM2 on asthma. The asthma symptoms were recorded, and airway hyper-responsiveness (AHR), expression levels of cytokines in the serum or bronchoalveolar lavage fluid (BALF), and the number of BALF leukocytes were evaluated. In addition, hematoxylin and eosin staining and immunohistochemistry of lung tissues was performed. The results demonstrated that PIM2 was overexpressed in patients with asthma in natural Treg cells. Inhibition of PIM2 attenuated asthma symptoms, and improved AHR and airway inflammation compared with asthmatic mice without inhibition of PIM2. In addition, expression levels of interleukin (IL)-10 and forkhead box protein 3 (FOXP3) in BALF were increased following PIM2 inhibition (IL-10, $470.3 \pm 21.78$ vs. $533.7 \pm 25.55 \mathrm{pg} / \mathrm{ml}, \mathrm{P}<0.05$;
\end{abstract}

Correspondence to: Professor Guochao Shi or Dr Wei Tang, Department of Pulmonary Medicine, Ruijin Hospital, School of Medicine, Shanghai Jiao Tong University, 197 Ruijin Er Road, Shanghai 200025, P.R. China

E-mail: shiguochao@hotmail.com

E-mail: tina_tangwei@163.com

${ }^{*}$ Contributed equally

Key words: asthma, proviral integration site for Moloney murine leukemia virus 2 , regulatory $\mathrm{T}$ cells, interleukin-10
FOXP3, $259 \pm 4.68$ vs. $279.3 \pm 3.68 \mathrm{pg} / \mathrm{ml}$; asthma and PIM2 inhibition groups, respectively; $\mathrm{P}<0.05$ ). In conclusion, PIM2 may exhibit an important role in asthma pathogenesis and exacerbate AHR, airway inflammation and asthma symptoms. These effects of PIM2 may be dependent on Treg cells and the secretion of IL-10 by Tregs. The results of the present study suggest that PIM2 may be a potential target molecule for asthma treatment.

\section{Introduction}

Asthma is a chronic, heterogeneous respiratory disease characterized by persistent airway inflammation and variable expiratory airflow limitation. It affects $1-18 \%$ of the global population and inhaled corticosteroids are the primary treatment (1). However, the mechanisms underlying the disease occurrence and progression remain to be fully elucidated. Type 2 inflammation, which is specifically caused by $\mathrm{T}$ helper (Th) 2 cells, is thought to have a central role in asthma pathogenesis, however, recently the importance of the dysregulation of other types of immune cells has also been emphasized, including Th17, Th9 and regulatory $\mathrm{T}$ (Treg) cells (2).

As a type of $\mathrm{CD}^{+} \mathrm{T}$ cell, Treg cells are essential for immune tolerance and the prevention of excessive inflammation. Tregs are divided into two subsets: Natural Treg cells (nTregs), which are derived from the thymus and account for the largest population of Treg cells in vivo; and inducible Treg cells (iTregs), which are derived from peripheral naïve $\mathrm{T}$ cells under a certain microenvironment (3). Forkhead box protein 3 (FOXP3) expression in both Treg cell subsets is crucial for their immune suppressive function (4). Interleukin (IL)-10 and transforming growth factor (TGF)- $\beta 1$ are the major cytokines that are produced by Treg cells. These cells limit the activation of proinflammatory cytokines, downregulate $\mathrm{T}$ cell-mediated inflammation and inhibit the proliferation of Th2 cells (5). The development of asthma is controlled by Treg cells as they inhibit the activation of Th2 cells, prevent the entry of effector immune cells into inflamed tissue, suppress IgE production and limit Th17-mediated inflammation (3), functions that are partially dependent on the cytokines secreted by Treg cells.

The proviral integration site for Moloney murine leukemia virus 2 (PIM2) is a type of serine/threonine kinase belonging to the PIM family, and has an important function in cellular proliferation, survival and differentiation (6). The majority of 
studies concerning PIM2 focus on its participation in hematologic malignancies and solid tumors, however, a number of studies have also indicated its role in regulating the immune system $(7,8)$. These studies demonstrated that PIM2 induced by FOXP3 is essential for Treg cell expansion and, conversely, PIM2 also inhibits the suppressive function of Treg cells by phosphorylating FOXP3, which indicates the complex roles of PIM2 in the regulation of Treg cells. As Treg cells have an important role in asthma pathogenesis, PIM2 may also influence the development of asthma inflammation, however, to the best of our knowledge, no previous studies have investigated the effect of PIM2 on asthma pathogenesis.

Therefore, the present study investigated the role of PIM2 in asthma pathogenesis, and the results demonstrated that PIM2 was overexpressed in peripheral blood mononuclear cells (PBMCs), and specifically in nTreg cells, from patients with asthma. In addition, inhibition of PIM2 in asthmatic mice alleviated airway inflammation, airway hyper-responsiveness (AHR) and associated symptoms, and these effects of PIM2 may be caused by decreased expression levels of FOXP3 and IL-10 in Treg cells.

\section{Patients and methods}

Subjects. The population consisted of patients with asthma ( $\mathrm{n}=12 ; 8$ males and 4 females) aged between 16 and 65 years, who were recruited from Ruijin Hospital (Shanghai, China) between January 2015 and September 2015. Healthy subjects $(n=8 ; 3$ male and 5 female) were included in the present study and were recruited as volunteers from Ruijin Hospital and School of Medicine, Shanghai Jiao Tong University (Shanghai, China). The diagnosis of asthma was made by the pulmonary physicians, according to the Global Initiative for Asthma guidelines (1). Subjects were excluded if they had experienced an asthma exacerbation in the previous 4 weeks or a respiratory infection 1 week prior to enrollment. All subjects gave written informed consent, and the study was approved by the Ethics Committee of Ruijin Hospital.

Sample preparation and $T$ cell isolation. Peripheral blood (20 $\mathrm{ml})$ was obtained in a sodium heparin vacuum tube. Subsequently, the blood sample was centrifuged $(700 \mathrm{x} \mathrm{g}$ for $20 \mathrm{~min}$ at $4^{\circ} \mathrm{C}$ ), the supernatant was discarded and the same volume of PBS was then added. The sample was added onto the surface of lymphocyte separation medium, Ficoll-Paque PLUS (GE Healthcare Life Sciences, Little Chalfont, UK), and centrifuged at $700 \mathrm{xg}$ for $20 \mathrm{~min}$ at $4^{\circ} \mathrm{C}$. PBMCs were collected from the middle layer and suspended at a density of $2 \times 10^{6}$ cells $/ \mathrm{ml}$ in RPMI-1640 medium with GlutaMAX (Gibco; Thermo Fisher Scientific, Inc., Waltham, MA, USA) supplemented with $100 \mathrm{U} / \mathrm{ml}$ penicillin, $100 \mu \mathrm{g} / \mathrm{ml}$ streptomycin and $10 \%$ heat inactivated fetal calf serum (Gibco; Thermo Fisher Scientific, Inc.). The cells were subsequently stained at $4^{\circ} \mathrm{C}$ for $30 \mathrm{~min}$ with the following fluorescently-labeled antibodies: CD4-fluorescein isothiocyanate (10 $\mu \mathrm{g} / \mathrm{ml}$; 11-0049-41; eBioscience; Thermo Fisher Scientific, Inc.), CD25-PerCP-Cyanine5.5 $(1.25 \mu \mathrm{g} / \mathrm{ml}$; 45-0259-42; eBioscience; Thermo Fisher Scientific, Inc.) and CD45RA-allophycocyanin (20 $\mu$ l/test; 550855; BD Biosciences, Franklin Lakes, NJ, USA) to sort for nTreg cells (CD4 ${ }^{+} \mathrm{CD} 25^{+}$ CD45RA- $\mathrm{T}$ cells) and naïve $\mathrm{T}$ cells $\left(\mathrm{CD} 4^{+} \mathrm{CD} 25^{-} \mathrm{CD}^{-} 5 \mathrm{RA}^{+}\right)$ on a FACS ARIA II cell sorter. Prior to cell staining, Fc receptors were blocked by incubating cells with $10 \%$ normal human serum (ImmunoReagents, Inc., Raleigh, NC, USA) for $20 \mathrm{~min}$ at $4^{\circ} \mathrm{C}$.

Induction of Th1, Th2 and iTreg cells. Naïve T cells $\left(1 \times 10^{6}\right.$ cells $\left./ \mathrm{ml}\right)$ were stimulated with CD3 and CD28 antibody-coated beads (Dynabeads Mouse T-activator; Invitrogen; Thermo Fisher Scientific, Inc.) at a 1:1 cell-to-bead ratio for 3 days at $37^{\circ} \mathrm{C}$ to induce $\mathrm{T}$ cell differentiation. Cultures were supplemented with IL-12 (10 ng/ml), IL-2 (50 U/ml) and anti-IL-4 (10 $\mu \mathrm{g} / \mathrm{ml} ; 556917)$ for Th1 induction, IL-4 $(5,000 \mathrm{U} / \mathrm{ml}), \mathrm{IL}-2(100 \mathrm{U} / \mathrm{ml})$, anti-interferon $\gamma(10 \mu \mathrm{g} / \mathrm{ml}$; $554699)$ and anti-IL-12 (10 $\mu \mathrm{g} / \mathrm{ml} ; 555065)$ for Th2 induction, and IL-2 (100 U/ml), TGF- $\beta 1(5 \mathrm{ng} / \mathrm{ml})$, anti-IL-4 $(10 \mu \mathrm{g} / \mathrm{ml})$, anti-IFN $\gamma(10 \mu \mathrm{g} / \mathrm{ml})$ and anti-IL-12 $(10 \mu \mathrm{g} / \mathrm{ml})$ for iTreg induction, at $37^{\circ} \mathrm{C}$ for 3 days. All antibodies and reagents were purchased from BD Biosciences.

RNA extraction and reverse transcription-quantitative polymerase chain reaction $(R T-q P C R)$. Total RNA in total PBMCs and individual T cell subsets was isolated with TRIzol reagent (Invitrogen; Thermo Fisher Scientific, Inc.) and was reverse transcribed into cDNA with Reverse Transcription system (Promega Corporation, Madison, WI, USA), according to manufacturer's protocol. Primers were designed by Invitrogen and synthesized by BioTNT (Shanghai, China), according to the manufacturer's protocol. For amplification, a SYBR-Green I qPCR kit was used (BioTNT). The thermocycling conditions were as follows: $95^{\circ} \mathrm{C}$ for $5 \mathrm{~min}$ followed by 40 cycles of $95^{\circ} \mathrm{C}$ for $5 \mathrm{sec}, 55^{\circ} \mathrm{C}$ for $20 \mathrm{sec}$ and $72^{\circ} \mathrm{C}$ for $20 \mathrm{sec}$. Each reaction was performed in triplicate on the ABI ViiA 7 Real-Time PCR system (Invitrogen; Thermo Fisher Scientific, Inc.) and expression was normalized using the $2^{-\Delta \Delta C q}$ method (9) to the expression of the housekeeping gene $\beta$-actin. The specific primers used were as follows: PIM2, 5'-ACTCCA GGTGGC CATCAAAG-3' (forward) and 5'-TCCATAGCA GTGCGA CTTCG-3' (reverse); and $\beta$-actin, 5'-AAGGTGACA GCAGTC GGTT-3' (forward) and 5'-TGTGTGGACTTGGGA GAG G-3' (reverse).

Immunocytochemistry. Human nTreg cells were obtained ( $1 \times 10^{4}-1 \times 10^{5}$ cells/sample) as aforementioned, fixed in $4 \%$ paraformaldehyde (PFA) for $15 \mathrm{~min}$ at room temperature, permeabilized with $1 \%$ Triton-X-100 for $5 \mathrm{~min}$ at room temperature and incubated with $3 \% \mathrm{H}_{2} \mathrm{O}_{2}$ for $20 \mathrm{~min}$ at room temperature. Cells were subsequently incubated with 5\% BSA (Sigma Aldrich; Merck KGaA, Darmstadt, Germany) for blocking at room temperature for $20 \mathrm{~min}$ and then anti-PIM2 mouse antibody $(0.5 \mu \mathrm{g} / \mathrm{ml}$; MAB4355; R\&D Systems, Inc., Minneapolis, MN, USA) at $4^{\circ} \mathrm{C}$ overnight. Following three washes with PBS, cells were incubated with rabbit anti-mouse peroxidase secondary antibody (1:200 dilution; A9044; Sigma Aldrich; Merck KGaA) for $2 \mathrm{~h}$ at room temperature. Following an additional three washes with PBS, cells were stained with a 3,3'-diaminobenzidine color developing reagent kit, and nuclei were stained with $1 \%$ hematoxylin for $2 \mathrm{~min}$ at room temperature. Samples were examined on a Nikon Eclipse 50i microscope (Nikon Corporation, Tokyo, Japan) and the images were analyzed with Image-Pro Plus 6.0 software (Media Cybernetics, Inc., Rockville, MD, USA). 
Animals and asthma model. A total of 90 Female BALB/c mice were purchased from Shanghai SLAC laboratory Co., Ltd. (Shanghai, China). Mice were aged between 6 and 8 weeks-old, weighing between 20 and $22 \mathrm{~g}$, and were housed at $18-25^{\circ} \mathrm{C}$, humidity $50-60 \%, 0.03 \% \mathrm{CO}_{2}, 12 / 12 \mathrm{~h}$ light/dark cycle and food/water were available ad libitum and refreshed every 3 days. The mice received an intraperitoneal injection of $100 \mu \mathrm{g}$ ovalbumin (OVA; Sigma Aldrich; Merck KGaA) and $2 \mathrm{mg}$ alum (Sigma Aldrich; Merck KGaA) in PBS on days 0,7 and 14. On days 25, 26 and 27 , the mice were challenge with aerosolized $1 \%$ OVA in PBS for $30 \mathrm{~min}$. Control animals received PBS intraperitoneally with alum on days 0,7 and 14 , and were challenged with aerosolized PBS on days 25, 26 and 27. The PIM2 inhibitor (5Z)-5-[[3-(Trifluoromethyl) phenyl] methylene]-2,4thiazolidinedione (Z)-5-(3-trifluoromethylbenzylidene) thiazolidine-2,4-dione (5 $\mu \mathrm{g} / \mu \mathrm{l}$; Sigma Aldrich; Merck KGaA) was given $30 \mathrm{~min}$ prior to challenge with OVA by intraperitoneal injection to OVA-sensitized mice on days 25, 26 and 27.

Evaluation of AHR and asthma symptoms. AHR was assessed by measuring changes of dynamic lung compliance (Cdyn) in response to increasing doses $(0-4 \mathrm{mg} / \mathrm{ml})$ of acetylcholine (Ach; Shanghai Mengry Biotechnology Co., Ltd., Shanghai, China) injected into the tail intravenously in anesthetized and ventilated mice. AHR was assessed using the AniRes2005 animal lung function analysis system (Beijing Bestlab High-Tech Co., Ltd., Beijing, China) $24 \mathrm{~h}$ after the last OVA challenge. Asthma symptoms, including cyanosis, frequently scratching the mouth/nose/limbs (>10 scratches in $5 \mathrm{sec}$ ) and standing upright were observed during challenge on days 25, 26 and 27. Time to each symptom occurrence (indicated as T1, T2 and T3) were measured and symptom scores of each mouse on different days was calculated as $[(30-\mathrm{T} 1)+(30-\mathrm{T} 2)+(30-\mathrm{T} 3)] / 3$.

Bronchoalveolar lavage fluid (BALF), lung histology and immunohistochemistry (IHC). Experiments were performed $24 \mathrm{~h}$ after the final OVA challenge. Lungs were lavaged with $1 \mathrm{ml}$ PBS through the trachea immediately following the assessment of AHR, and the number of total leukocytes was counted with a hemocytometer, and cell differentiation was performed on cytospin slides prepared with Wright-Giemsa staining to differentiate between leukocytes. Cells were seeded in PBS medium (Beyotime Institute of Biotechnology, Haimen, China) at $1 \times 10^{5}$ cells $/ \mathrm{ml}$ and stained with Fast Wright and Giemsa Stain kit (Nanjing Jiancheng Technology Co., Ltd., Nanjing, China), according to manufacturer's protocol. Percentages of each cell were counted per sample with a light microscope: Number of each cell type/every 200 cells x $100 \%$.

The left upper lobes of the lungs were fixed in $2 \mathrm{ml} 4 \%$ PFA at $4^{\circ} \mathrm{C}$ for $12-24 \mathrm{~h}$ and embedded in paraffin. Prepared sections $(4 \mu \mathrm{m})$ were stained with $0.5 \%$ hematoxylin (10 $\mathrm{min})$ and $0.5 \%$ eosin $(30 \mathrm{sec})$ at room temperature using standardized protocols and analyzed with a Nikon Eclipse $50 \mathrm{i}$ microscope. IHC was performed on $4 \mu \mathrm{m}$-PFA fixed sections. Antigen retrieval was performed with $0.01 \mathrm{M}$ citric acid buffer ( $\mathrm{pH} \mathrm{6.0)}$ ) for $15 \mathrm{~min}$ at $95^{\circ} \mathrm{C}$ prior to rehydration in descending alcohol series. Sections were subsequently blocked with 5\% goat serum (Beyotime) for $20 \mathrm{~min}$ at room temperature. Endogenous peroxidase/phosphatase activity was blocked with $3 \%$ hydrogen peroxide prior to incubation overnight at $4^{\circ} \mathrm{C}$ with anti-PIM2 $(2 \mu \mathrm{g} / \mathrm{ml}$; sc13514; Santa Cruz Biotechnology, Inc., Dallas, TX, USA) and anti-FOXP3 (1:500; SAB5300461; Sigma-Aldrich; Merck KGaA). Subsequently, sections were incubated for $30 \mathrm{~min}$ at $37^{\circ} \mathrm{C}$ with anti-mouse IgG-peroxidase secondary antibody (1:100; A0168; Sigma Aldrich; Merck KGaA). 3,3'-Diaminobenzidine was applied for chromogen detection. Sections were counterstained with $0.5 \%$ hematoxylin and eosin for $30 \mathrm{sec}$ at room temperature and visualized with the Nikon Eclipse 50i upright microscope (magnification, x200). Lung inflammation was scored as described previously (10). Expression levels of PIM2 and FOXP3 in lung tissues were evaluated by Image-Pro Plus 6.0, and recorded as the mean optical density.

ELISA. Experiments were performed $24 \mathrm{~h}$ after the last OVA challenge. A total of $0.5-0.8 \mathrm{ml}$ blood was drawn from each mouse. Serum was collected following centrifugation at $1,000 \mathrm{x} \mathrm{g}$ for $5 \mathrm{~min}$ at $4^{\circ} \mathrm{C}$. Concentrations of cytokines and OVA specific IgE in the BALF and serum of mice were measured by ELISA, according to the protocols of each ELISA kit (FOXP3, MR45686; IL-4, MR63901; IL-5, MR63900; TRG- $\beta$, MR63871; IL-10, MR63912; OVA specific IgE, MR64027; Shanghai Mengry Biotechnology Co., Ltd.).

Statistical analysis. Data are presented as the mean \pm standard deviation for continuous variants. All analyses were performed using SPSS (version 20; IBM Corp., Armonk, NY, USA). Student's t-test was used to determine differences between two groups. For comparisons between multiple groups, one-way analysis of variance (post hoc Tukey test) was used. Nonparametric analyses, using the Mann-Whitney U test or Kruskal-Wallis test (post hoc Dunn's test), were applied if the distributions of numerical data were not normal. Comparisons were made using Pearson's Chi-square test if the data were categorical variants. $\mathrm{P}<0.05$ was considered to indicate a statistically significant difference.

\section{Results}

Baseline characteristics of subjects. Asthma patients $(n=12)$ and healthy subjects $(n=8)$ were included in the present study. The age of the asthma patients was $47 \pm 10$ years, with no significant difference compared with healthy controls $(38 \pm 9$ years; $\mathrm{P}>0.05)$. A total of 8 patients with asthma were male, which was comparable to healthy subjects ( 3 males; $\mathrm{P}>0.05$ ).

PIM2 is highly expressed in asthma patients and primarily located in Treg cells. PIM2 has been reported to be expressed ubiquitously, with the highest levels in the brain and lymphoid tissues (11). In addition, PIM2 is established to be upregulated in various hematologic malignancies, including acute or chronic myeloid leukemia, acute lymphoblastic leukemia, multiple myeloma and lymphoma (12), however, its expression pattern in human T lymphocytes is not clear. The present study isolated naïve $\mathrm{T}$ cells and nTreg cells from healthy human PBMCs and induced naïve T cells into Th1, Th2 and iTreg cells by different culture conditions, the results demonstrated that PIM2 was primarily expressed in Treg cells, particularly nTreg cells (Fig. 1A). 
A

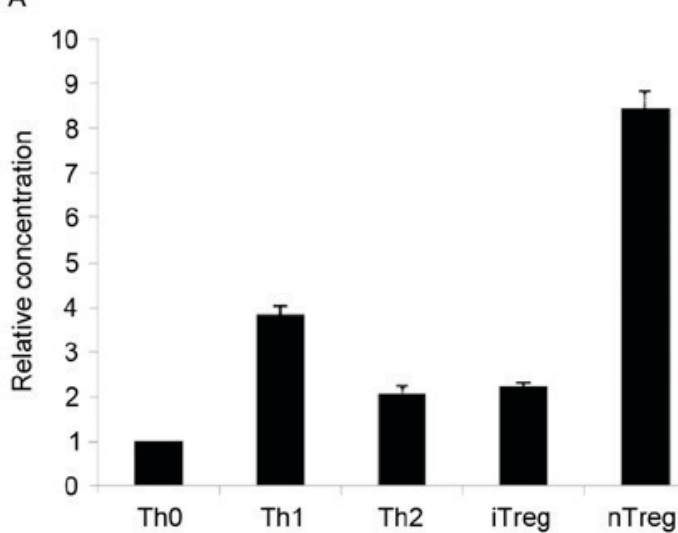

C

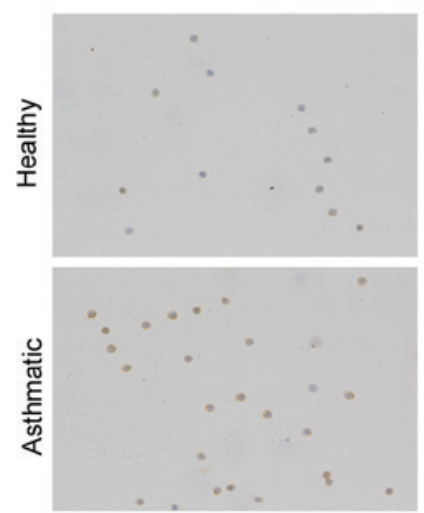

B

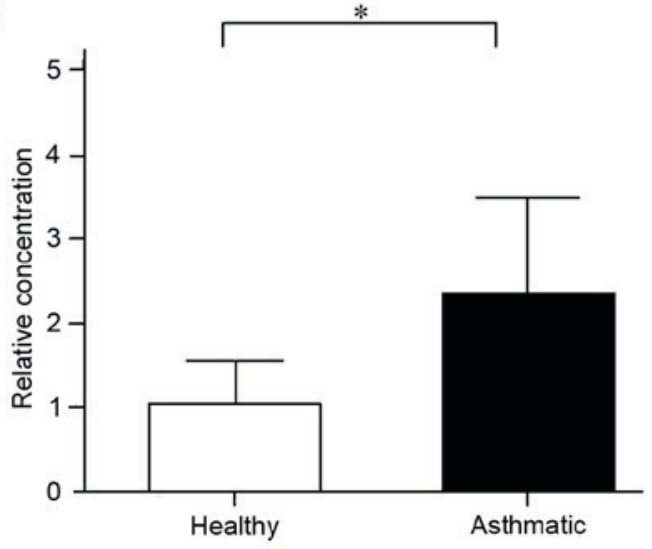

D

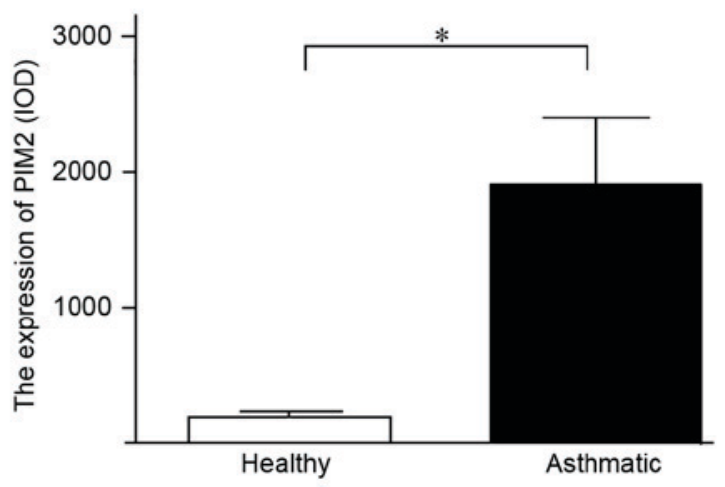

Figure 1. The expression of PIM2 in PBMCs and Treg cells from patients with asthma was higher compared with healthy subjects. (A) Expression levels of PIM2 mRNA in different types of T cells in healthy subjects. (B) Expression level of PIM2 mRNA in PBMCs. (C) Representative images (magnification, $\mathrm{x} 200$ ) of PIM2 expression in nTreg cells from patients with asthma and healthy subjects, as measured by immunocytochemistry. (D) Quantified PIM2 expression levels in Treg cells, as measured by immunocytochemistry. " $\mathrm{P}<0.05$, as indicated. PIM2, proviral integration site for Moloney murine leukemia virus 2 ; PBMCs, peripheral blood mononuclear cells; Tregs, T regulatory cells; Th, T helper cells; iTregs, inducible Tregs; nTregs, natural Tregs; IOD, integrated optical density.

To the best of our knowledge, no previous study has investigated the PIM2 expression level in patients with asthma, and the present study demonstrated that the mRNA and protein expression levels of PIM2 in PBMCs and nTregs, respectively, were higher in patients with asthma compared with healthy subjects (Fig. 1B-D), indicating that PIM2 may have an important role in asthma pathogenesis.

PIM2 is essential for airway inflammation and AHR. As PIM2 was highly expressed in patients with asthma, an asthma mouse model and PIM2 inhibitor were used to investigate the role of PIM2 in asthma pathogenesis. The mice were sensitized and challenged with OVA, and the PIM2 inhibitor was added during the challenge period. Serum levels of PIM2 were determined in control, OVA and PIM2 inhibitor mice, and levels were significantly increased in the OVA treatment group compared with the control group, while levels were significantly decreased in the OVA + PIM2 inhibitor group compared with the OVA-only group (Fig. 2A). In addition, the serum levels of OVA-specific IgE were measured, with levels significantly increased in the OVA group compared with controls. However, although PIM2 inhibitor reduced the levels compared with the OVA-only group, this reduction was not statistically significant (Fig. 2B). Furthermore, the results indicated that PIM2 inhibition improved AHR (indicated as Cdyn) and asthma-associated symptoms significantly, compared with OVA-treated mice only (Fig. 2C and D).

Additionally, increases in BALF cell numbers induced by OVA were marginally decreased following PIM2 inhibition (Fig. 2E), and the percentages of BALF eosinophils and neutrophils were significantly reduced following PIM2 inhibition in OVA-treated mice (Fig. 2F). In addition, H\&E staining of lung tissues from mouse models demonstrated that PIM2 inhibition alleviated airway inflammation compared with the OVA-only group (Fig. 3A and B). These results indicate an important role of PIM2 in inducing airway inflammation and AHR, however, the underlying mechanism was yet to be determined.

PIM2 inhibits $I L-10$ production by regulating FOXP3. A previous study demonstrated the role of PIM2 in regulating the function of Treg cells by mediating FOXP3 phosphorylation (8), however, its influence on FOXP3 expression and downstream cytokines, including TGF- $\beta 1$ and IL-10, in asthma mouse models has not previously been investigated. The present study demonstrated that PIM2 inhibition led to increased expression levels of FOXP3 in lung tissues (Fig. 3A and $\mathrm{C}$ ) and BALF compared with OVA-only treated mice (Fig. 4A), indicating that PIM2 may downregulate FOXP3 expression in allergic asthma. 
A

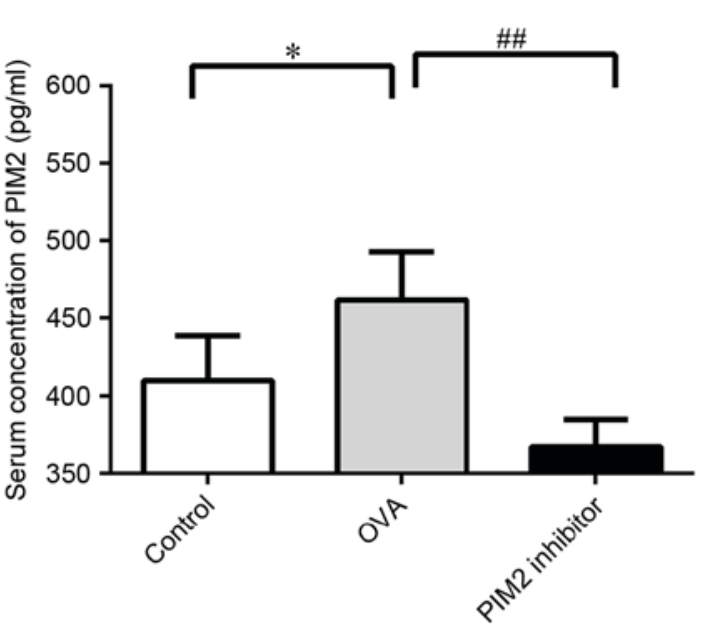

C

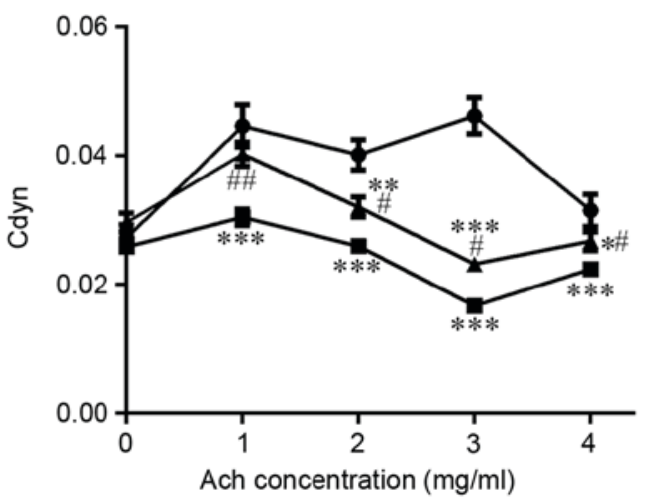

$E$

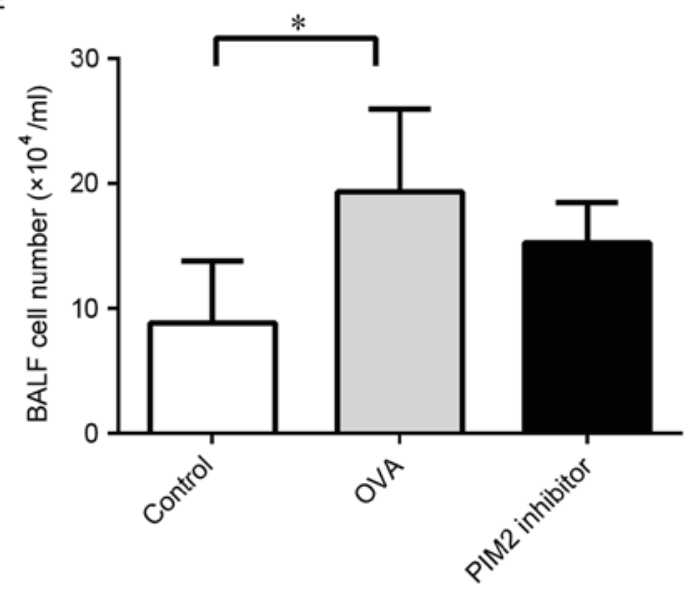

B

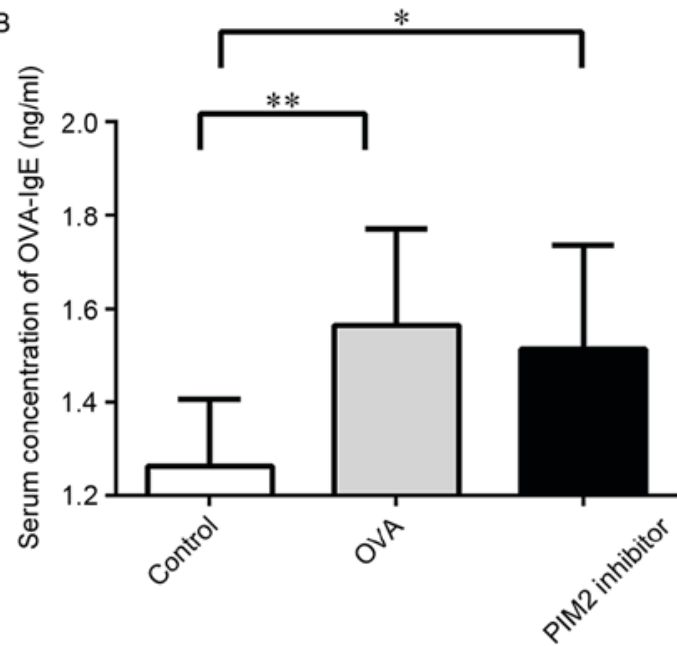

D

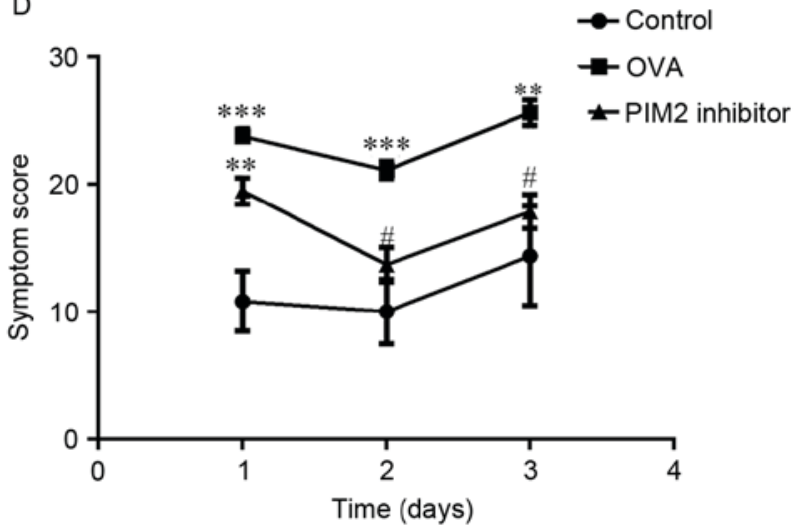

$\mathrm{F}$

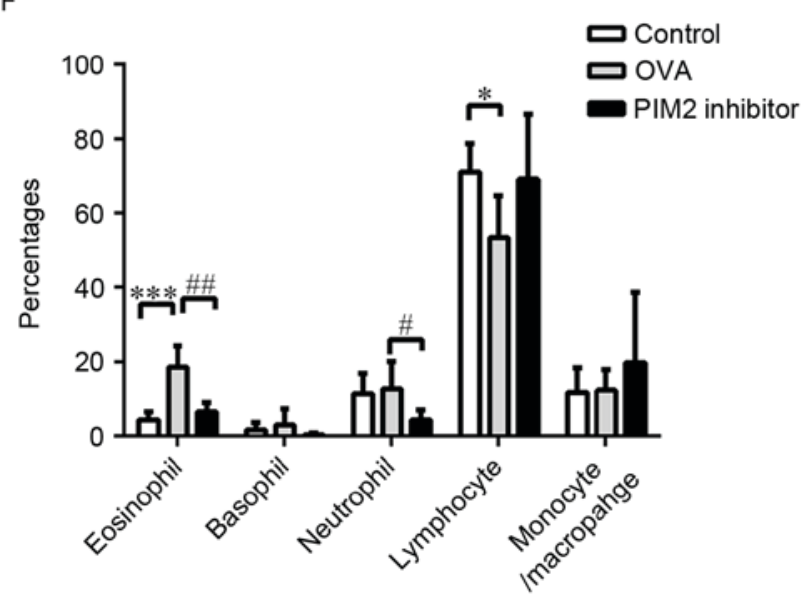

Figure 2. PIM2 inhibition resulted in attenuated asthma symptoms, improved airway hyper-responsiveness and reduced airway-infiltrated eosinophils and neutrophils in asthmatic mice. Serum concentrations of (A) PIM2 and (B) OVA-specific IgE were determined in the indicated groups. (C) Changes in lung compliance, indicated as Cdyn, in response to increased doses of Ach. (D) Asthma symptom score during the 3 challenge days. (E) Total number of leukocytes in BALF. (F) Percentages of different leukocyte types in BALF. ${ }^{*} \mathrm{P}<0.05,{ }^{* * *} \mathrm{P}<0.01$ and ${ }^{* * * *} \mathrm{P}<0.001$ vs. control group; ${ }^{\#} \mathrm{P}<0.05$ and ${ }^{\# \#} \mathrm{P}<0.01$ vs. OVA group. $\mathrm{n} \geq 5$ per group. PIM2, proviral integration site for Moloney murine leukemia virus 2; OVA, ovalbumin; Cdyn, dynamic lung compliance; Ach, acetylcholine; BALF, bronchoalveolar lavage fluid.

Additionally, BALF and serum IL-10 levels were increased following PIM2 inhibition in OVA-treated mice (Fig. 4B and C), however, the expression levels of TGF- $\beta 1$ in BALF and serum were not altered by PIM2 inhibition in asthmatic mice (Fig. 4D and E). These results indicate that PIM2 may inhibit IL-10 production, and not TGF- $\beta 1$ production, to suppress FOXP3 expression. Additionally, the expression levels of Th2 cytokines in the BALF and serum, including IL-4 and IL-5, were not altered following PIM2 inhibition in OVA-treated mice (Fig. 5).

\section{Discussion}

PIM2 has been primarily recognized as an oncogene since its discovery, however, its role in regulating the immune system has not been extensively investigated. The present study 
A
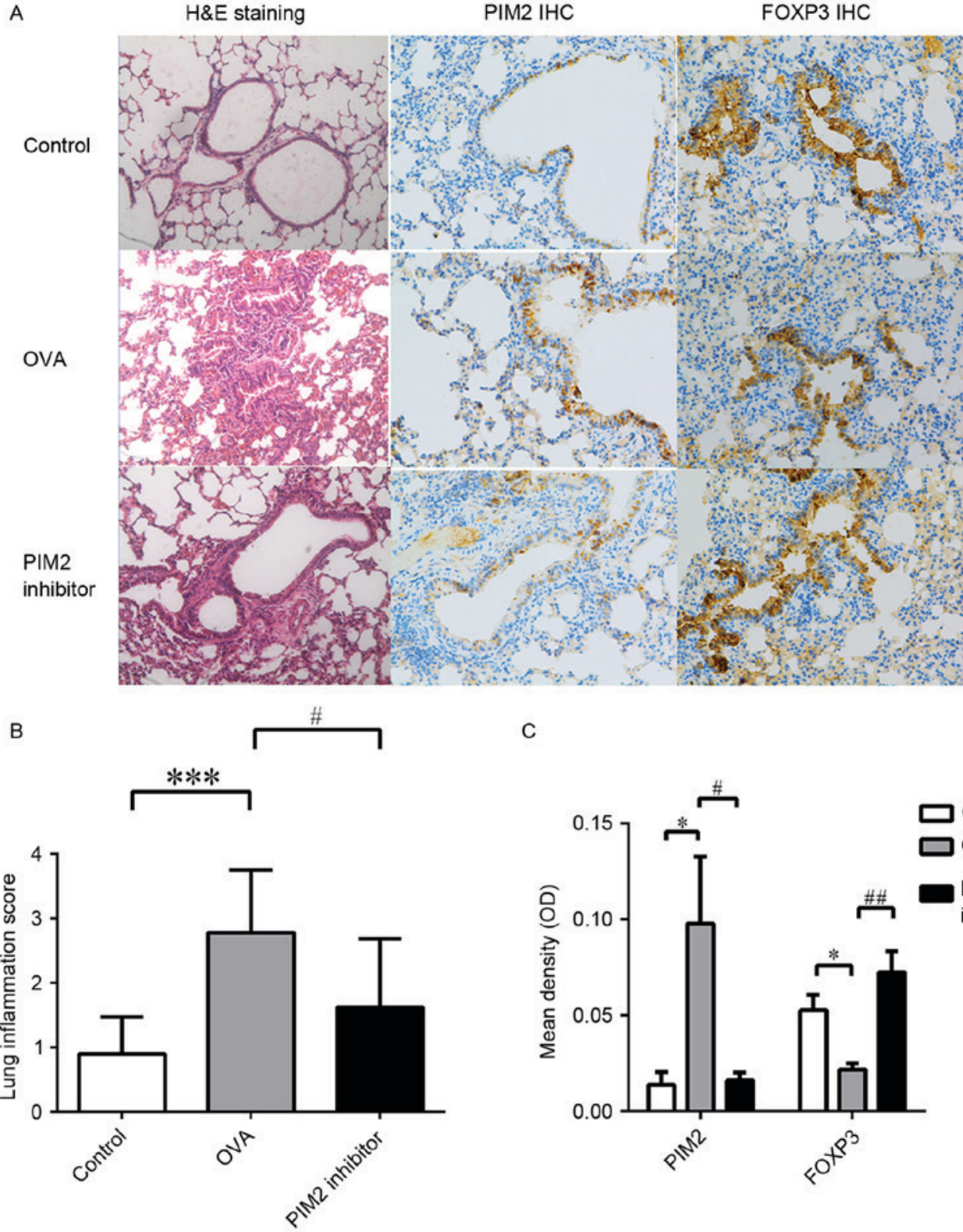

C

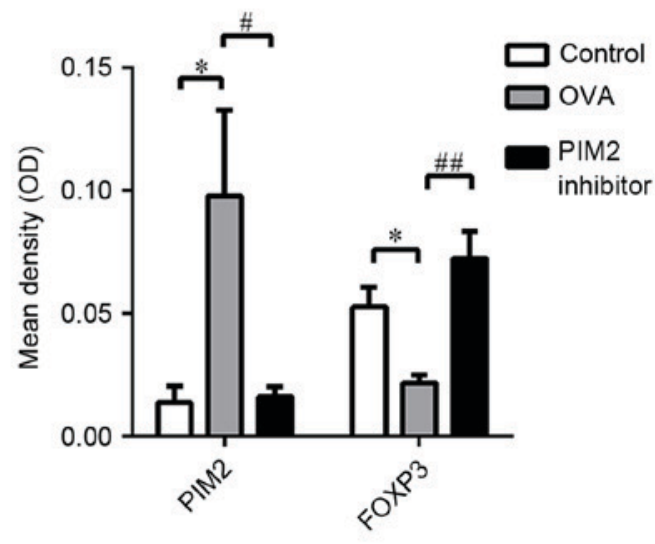

Figure 3. H\&E staining and IHC of lung tissues from each group. (A) Representative images of lung tissue following H\&E staining (magnification, x100) and IHC (magnification, x200) for PIM2 and FOXP3. (B) Quantitative results of H\&E staining evaluating lung tissue inflammation. (C) Quantitative results of IHC for PIM2 and FOXP3. ${ }^{*} \mathrm{P}<0.05$ and ${ }^{* * *} \mathrm{P}<0.001$ vs. control group; ${ }^{*} \mathrm{P}<0.05$ and ${ }^{\# \#} \mathrm{P}<0.01$ vs. OVA group. $\mathrm{n} \geq 5$ per group. H\&E, hematoxylin and eosin; IHC, immunohistochemistry; PIM2, proviral integration site for Moloney murine leukemia virus 2; FOXP3, forkhead box protein 3; OVA, ovalbumin; OD, optical density.

investigated its role in asthma development and demonstrated that PIM2 may have an important role in asthma pathogenesis. In patients with asthma, PIM2 expression levels were elevated, particularly in nTreg cells. Furthermore, results from an animal model of asthma further confirmed the critical role of PIM2 in promoting airway inflammation (both eosinophilic and neutrophilic) and AHR, which may occur via the regulation of FOXP3 and IL-10 expression in Treg cells.

Although no previous study has elucidated the role of PIM2 in asthma pathogenesis, the following observations have indicated the potential of a role for PIM2 in asthma: PIM2 is induced upon IL-4 release to promote $\mathrm{T}$ cell growth and survival, indicating its role in regulating adaptive immune system (8); it has been reported that PIM1, which is $61 \%$ homologous to PIM2 (11), has the ability to affect airway inflammation, AHR and the production of type 2 cytokines in asthmatic mice, indicating that PIM2 may exhibit a similar effect on asthma development; PIM2 has been recognized as a molecule that may lead to mammalian target of rapamycin complex 1 (mTORC1) activation, which inhibits Treg differentiation $(7,13,14)$; and, conversely, the inhibition of PIM2 was demonstrated to enhance the function of Treg cells in vitro (8). It is established that Treg cells are important regulators in excessive immune responses, such as persistent airway inflammation in patients with asthma, and these reports have indicated that PIM2 may have an important function in asthma pathogenesis by regulating the proliferation, differentiation and function of Treg cells.

Overexpression of PIM2 is commonly reported in hematologic and solid malignancies, including acute or chronic leukemia, lymphomas, and prostate and liver cancers, however, no previous study has measured its expression level in patients 


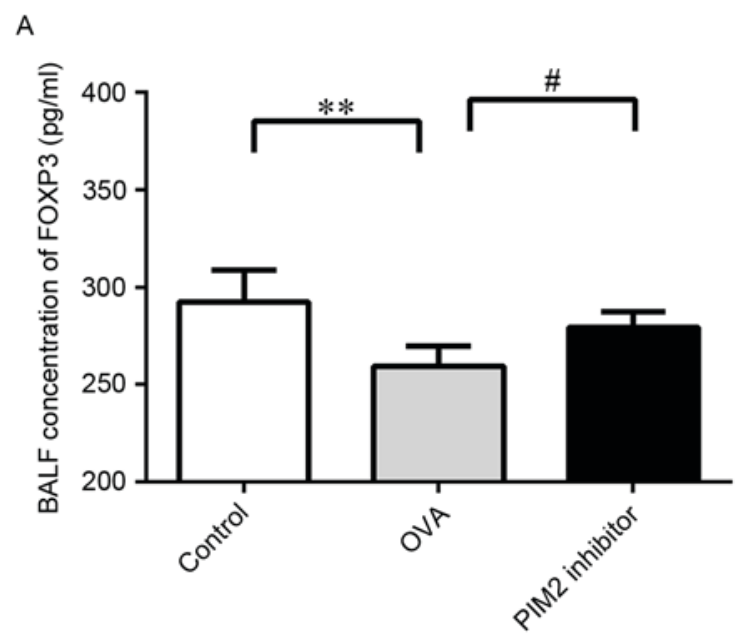

B

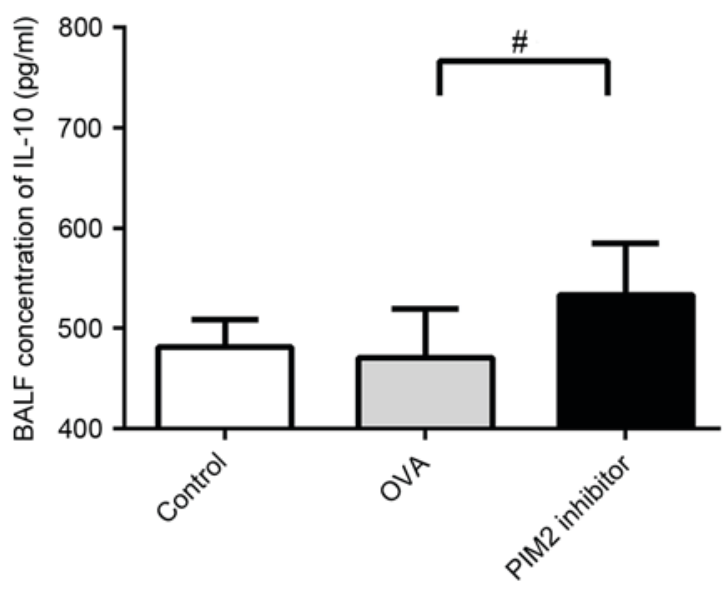

D

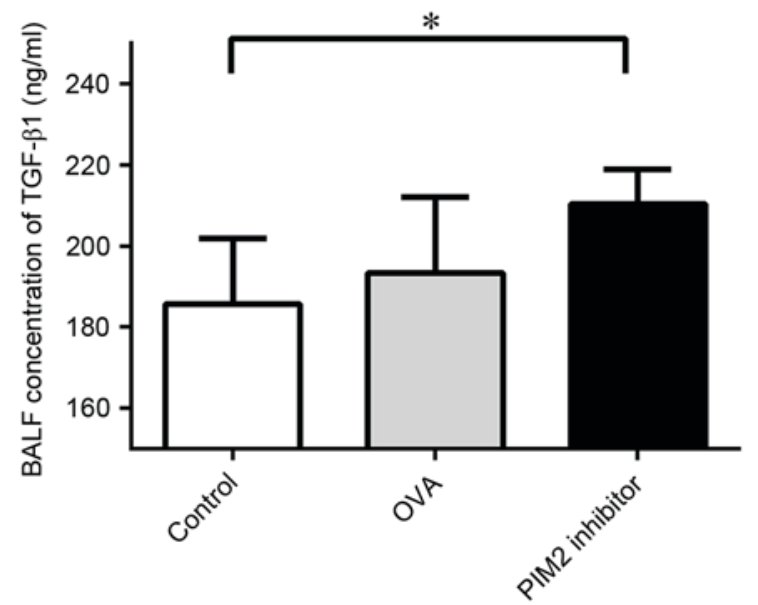

C

$E$
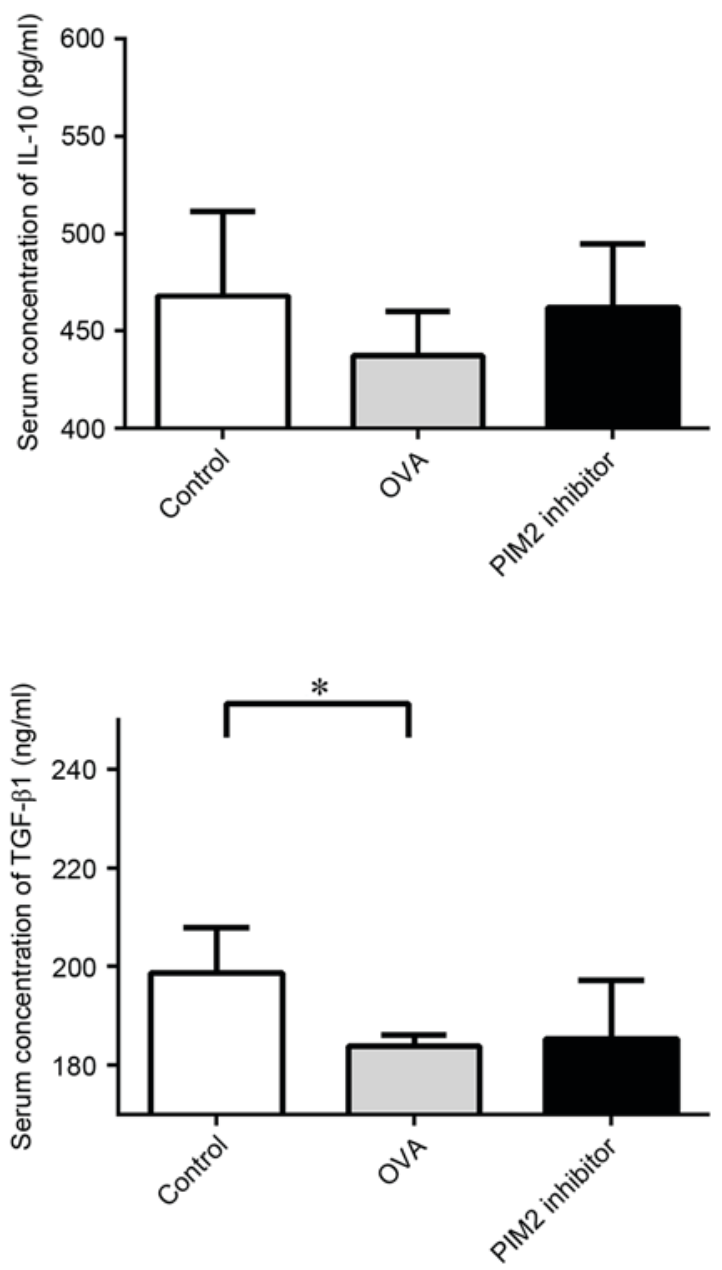

Figure 4. PIM2 inhibition increased the expression levels of FOXP3 and IL-10. (A) BALF concentrations of FOXP3 in each group. Concentration of IL-10 in (B) BALF and (C) serum in each group. Concentration of TGF- $\beta 1$ in (D) BALF and (E) serum in each group. " $\mathrm{P}<0.05$ and ${ }^{*} \mathrm{P}<0.01$ vs. control group; ${ }^{*} \mathrm{P}<0.05$ vs. OVA group. $\mathrm{n} \geq 5$ per group. PIM2, proviral integration site for Moloney murine leukemia virus 2; FOXP3, forkhead box protein 3; IL, interleukin; BALF, bronchoalveolar lavage fluid; TGF, transforming growth factor; OVA, ovalbumin.

with asthma (11,15). In healthy subjects, PIM2 is constitutively expressed in lymphoid cells $(16,17)$, and a previous report demonstrated high expression of PIM2 in nTreg cells (18). Therefore, the present study collected PBMCs from asthmatic and healthy subjects in order to measure the difference in PIM2 expression level between asthmatic and healthy subjects. The results demonstrated that PIM2 was overexpressed in patients with asthma compared with healthy subjects in nTreg cells, which was consistent with our hypothesis that PIM2 may influence asthma development by regulating Treg cells.

To further confirm our hypothesis, animal experiments were performed. The results demonstrated that PIM2 inhibition alleviated airway inflammation and AHR, and reversed the downregulated FOXP3 and IL-10 expression levels in 
A

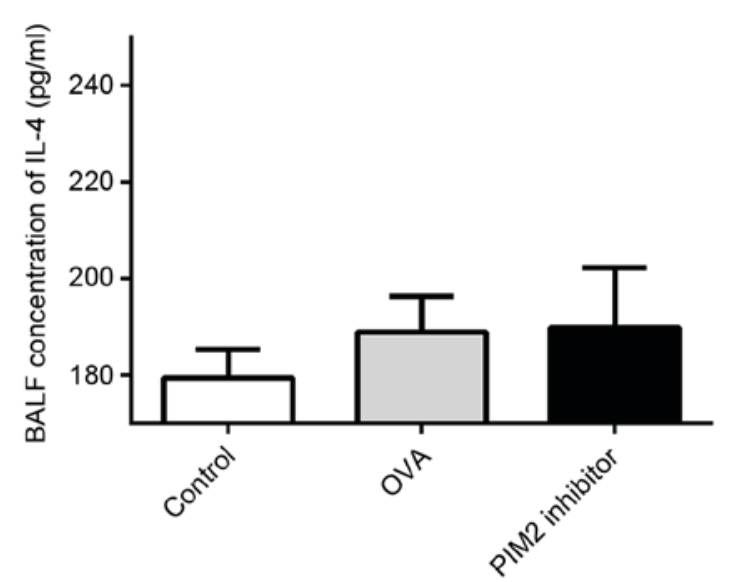

C

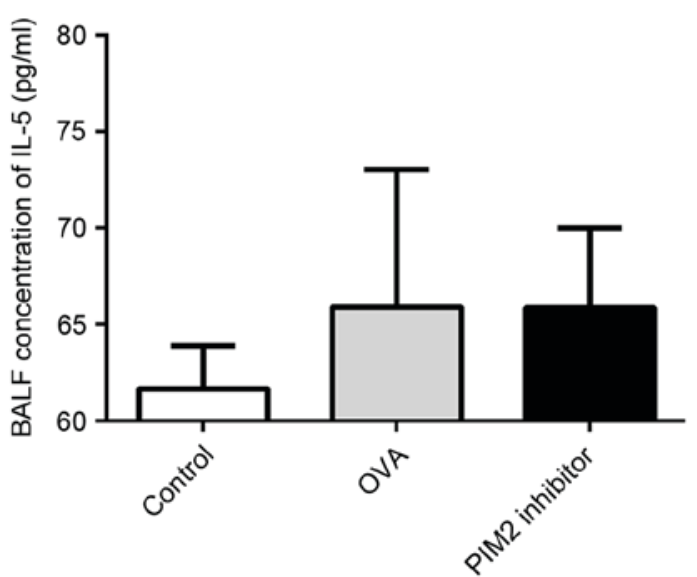

B

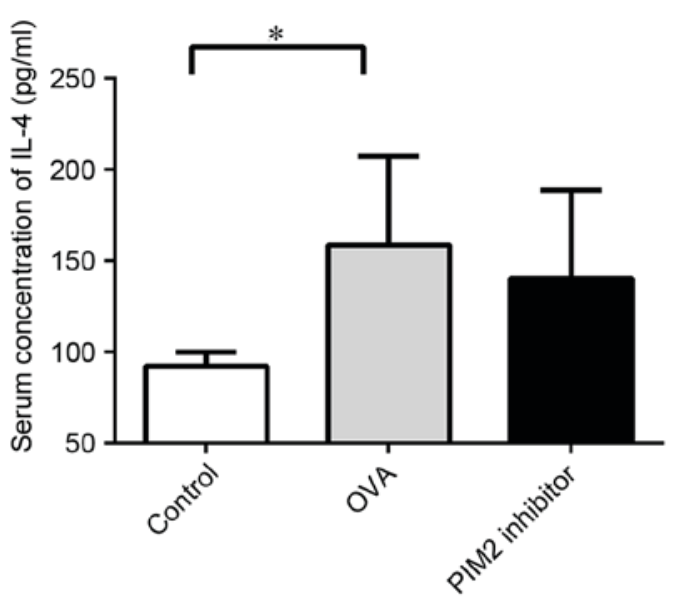

D

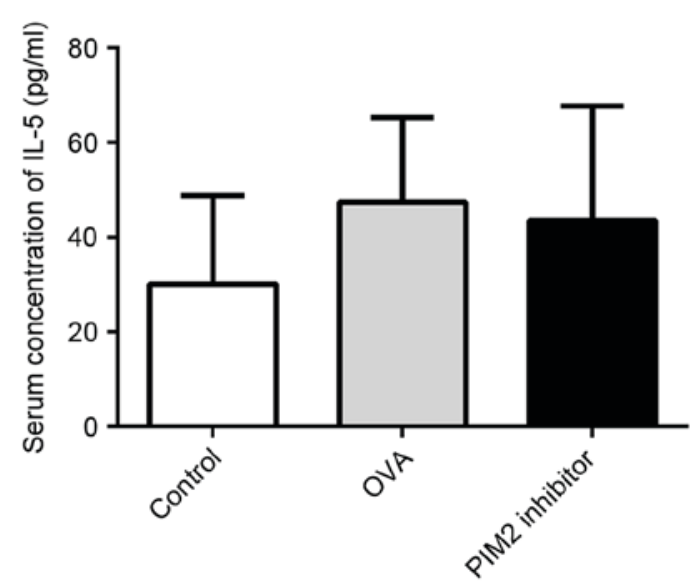

Figure 5. Expression of IL-4 and IL-5 was not affected by PIM2 inhibition in asthmatic mice. Concentration of IL-4 in (A) BALF and (B) serum in each group. Concentration of IL-5 in (C) BALF and (D) serum in each group. ${ }^{*} \mathrm{P}<0.05$ vs. control group. $\mathrm{n} \geq 5$ per group. IL, interleukin; PIM2, proviral integration site for Moloney murine leukemia virus 2; BALF, bronchoalveolar lavage fluid; OVA, ovalbumin.

asthmatic mice. Unlike PIM1, which is associated with the survival of eosinophils, PIM2 has not been previously reported to influence eosinophil survival in vitro or in vivo (19). Therefore, the reduced percentage of eosinophils in BALF following PIM2 inhibition in asthmatic mice may be the result of enhanced Treg cell function or cytokine secretion, which may inhibit the function of type 2 cytokines, including IL-4 and IL-5, but not the expression levels of these cytokines, as PIM2 inhibition appeared to exert no direct influence on the expression levels of IL-4 and IL-5 in the present study.

Although the association between PIM2 and Treg cells is established, the molecular mechanisms underlying this association remain unclear. A previous study reported that PIM2 suppressed the function of Treg cells by phosphorylating the FOXP3 N-terminal domain (8), however, this may be only one of several mechanisms. For example, PIM2 is also reported to upregulate mTORC expression (20), which has a negative effect on Treg cell function and differentiation (4). In addition, increased expression of PIM2 was also demonstrated to be associated decreased phosphatase and tensin homolog deleted on chromosome 10, which is highly expressed in Treg cells and regulates their differentiation $(4,21)$. The present study has demonstrated that PIM2 may influence the expression of FOXP3 and IL-10, both of which represent the function of Treg cells, however, the precise underlying molecular mechanisms behind this phenomenon remain unclear, and further studies are required. In addition, previous studies have indicated the important role of TGF- $\beta 1$ in asthma pathogenesis and airway remodeling $(22,23)$, however, in the current study, its expression level was increased marginally in BALF and decreased significantly in the serum of asthmatic mice compared with the control group. This result may be due to relative acute and early phase of asthma in this mouse model, in which airway remodeling was not predominant. Increased eosinophils in the airway may secret small amounts of this cytokine leading to the increased expression of this cytokine observed in the BALF. Conversely, systemic functional deficiency of Treg cells may be responsible for the reduced secretion of TGF- $\beta 1$ observed in the serum.

In conclusion, the present study is the first to demonstrate the critical role of PIM2 in asthma pathogenesis, and this effect may be dependent on Treg cells and the secretion of IL-10 by Tregs, which suppresses excessive airway inflammation in patients with asthma. As the number of studies that have investigated the association between asthma pathogenesis and PIM2 is limited, further studies should be performed to determine the mechanisms underlying the association and any therapeutic potential of this kinase. 


\section{Acknowledgements}

The present study was funded by Shanghai Municipal Education Commission (grant no. 14ZZ107) and the National Natural Science Foundation of China (grant nos. 81270083 and 81470216). The abstract was presented as the American Thoracic Society 2016 International Conference May 13-18, 2016 in San Francisco, CA and published as abstract no. A6701 in the American Journal of Respiratory and Critical Care Medicine 193, 2016.

\section{References}

1. From the global strategy for asthma management and prevention, global initiative for asthma (GINA) 2015. http://ginasthma.org/ wp-content/uploads/2016/01/GINA_Report_2015_Aug11-1.pdf. Accessed December, 2015.

2. Hirahara $\mathrm{K}$ and Nakayama T: $\mathrm{CD} 4^{+} \mathrm{T}$-cell subsets in inflammatory diseases: Beyond the Th1/Th2 paradigm. Int Immunol 28: 163-171, 2016.

3. Stelmaszczyk-Emmel A: Regulatory $\mathrm{T}$ cells in children with allergy and asthma: It is time to act. Respir Physiol Neurobiol 209: 59-63, 2015.

4. Kasper IR, Apostolidis SA, Sharabi A and Tsokos GC: Empowering regulatory T cells in autoimmunity. Trends Mol Med 22: 784-797, 2016.

5. Qiao YC, Shen J, Hong XZ, Liang L, Bo CS, Sui Y and Zhao HL: Changes of regulatory $\mathrm{T}$ cells, transforming growth factor-beta and interleukin-10 in patients with type 1 diabetes mellitus: A systematic review and meta-analysis. Clin Immunol 170: 61-69, 2016.

6. Uddin N, Kim RK, Yoo KC, Kim YH, Cui YH, Kim IG, Suh Y and Lee SJ: Persistent activation of STAT3 by PIM2-driven positive feedback loop for epithelial-mesenchymal transition in breast cancer. Cancer Sci 106: 718-725, 2015.

7. Yin G, Li Y, Yang M, Cen XM and Xie QB: Pim-2/mTORC1 pathway shapes inflammatory capacity in rheumatoid arthritis synovial cells exposed to lipid peroxidations. Biomed Res Int 2015: 240210, 2015

8. Deng G, Nagai Y, Xiao Y, Li Z, Dai S, Ohtani T, Banham A, Li B, Wu SL, Hancock W, et al: Pim-2 kinase influences regulatory $\mathrm{T}$ cell function and stability by mediating Foxp3 protein N-terminal phosphorylation. J Biol Chem 290: 20211-20220, 2015.

9. Livak KJ and Schmittgen TD: Analysis of relative gene expression data using real-time quantitative PCR and the 2(-Delta Delta C(T)) method. Methods 25: 402-408, 2001.

10. Massoud AH, Charbonnier LM, Lopez D, Pellegrini M, Phipatanakul W and Chatila TA: An asthma-associated IL4R variant exacerbates airway inflammation by promoting conversion of regulatory T cells to TH17-like cells. Nat Med 22: 1013-1022, 2016.
11. Narlik-Grassow M, Blanco-Aparicio C and Carnero A: The PIM family of serine/threonine kinases in cancer. Med Res Rev 34: 136-159, 2014.

12. Keeton EK, McEachern K, Dillman KS, Palakurthi S, Cao Y, Grondine MR, Kaur S, Wang S, Chen Y, Wu A, et al: AZD1208, a potent and selective pan-Pim kinase inhibitor, demonstrates efficacy in preclinical models of acute myeloid leukemia. Blood 123: 905-913, 2014.

13. Li MO and Rudensky AY: T cell receptor signalling in the control of regulatory T cell differentiation and function. Nat Rev Immunol 16: 220-233, 2016.

14. Zhang XH, Yu HL, Wang FJ, Han YL and Yang WL: Pim-2 modulates aerobic glycolysis and energy production during the development of colorectal tumors. Int J Med Sci 12: 487-493, 2015.

15. Alvarado Y, Giles FJ and Swords RT: The PIM kinases in hematological cancers. Expert Rev Hematol 5: 81-96, 2012.

16. Yang J, Li X, Hanidu A, Htut TM, Sellati R, Wang L, Jiang H and Li J: Proviral integration site 2 is required for interleukin-6 expression induced by interleukin-1, tumour necrosis factor- $\alpha$ and lipopolysaccharide. Immunology 131: 174-182, 2010.

17. Brault L, Gasser C, Bracher F, Huber K, Knapp S and Schwaller J: PIM serine/threonine kinases in the pathogenesis and therapy of hematologic malignancies and solid cancers. Haematologica 95: 1004-1015, 2010

18. Basu S, Golovina T, Mikheeva T, June CH and Riley JL: Cutting edge: Foxp3-mediated induction of pim 2 allows human T regulatory cells to preferentially expand in rapamycin. J Immunol 180: 5794-5798, 2008

19. Shin YS, Takeda K, Shiraishi Y, Jia Y, Wang M, Jackson L, Wright AD, Carter L, Robinson J, Hicken E and Gelfand EW: Inhibition of Pim1 kinase activation attenuates allergen-induced airway hyperresponsiveness and inflammation. Am J Respir Cell Mol Biol 46: 488-497, 2012

20. Ezell SA, Wang S, Bihani T, Lai Z, Grosskurth SE, Tepsuporn S, Davies BR, Huszar D and Byth KF: Differential regulation of mTOR signaling determines sensitivity to AKT inhibition in diffuse large B cell lymphoma. Oncotarget 7: 9163-9174, 2016.

21. Asanuma S, Tanaka J, Sugita J, Kosugi M, Shiratori S, Wakasa K, Shono Y, Shigematsu A, Kondo T, Kobayashi T, et al: Expansion of CD4(+) CD25(+) regulatory T cells from cord blood CD4(+) cells using the common $\gamma$-chain cytokines (IL-2 and IL-15) and rapamycin. Ann Hematol 90: 617-624, 2011.

22. Ojiaku CA, Yoo EJ and Panettieri RA Jr: Transforming growth factor $\beta 1$ function in airway remodeling and hyperresponsiveness. The missing link? Am J Respir Cell Mol Biol 56: 432-442, 2017.

23. Koćwin M, Jonakowski M, Przemecka M, Zioło J, Panek M and Kuna P: The role of the TGF-SMAD signalling pathway in the etiopathogenesis of severe asthma. Pneumonol Alergol Pol 84: 290-301, 2016. 